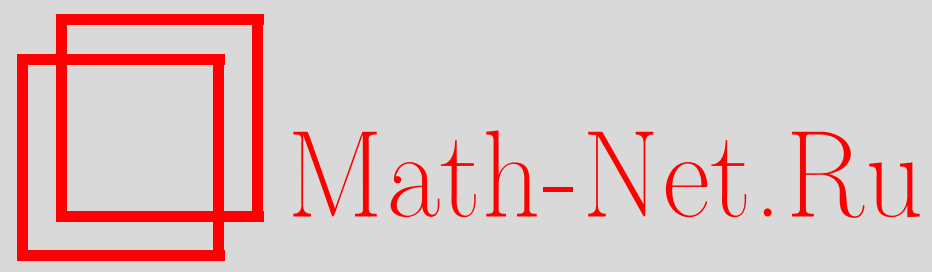

А. М. Рахматуллаев, У. А. Розиков, Гиббсовские меры и марковские случайные поля с отношением I, Матем. заметки, 2002, том 72, выпуск 1, 94-101

DOI: https://doi.org/10.4213/mzm407

Использование Общероссийского математического портала Math-Net.Ru подразумевает, что вы прочитали и согласны с пользовательским соглашением http://www.mathnet.ru/rus/agreement

Параметры загрузки:

IP : 54.196 .121 .252

26 апреля 2023 г., $15: 18: 36$

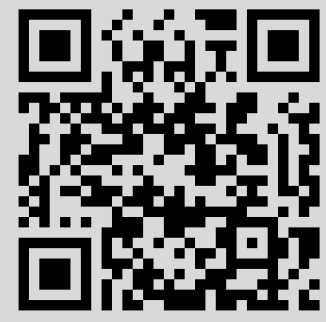




\title{
ГИББСОВСКИЕ МЕРЫ И МАРКОВСКИЕ СЛУЧАЙНЫЕ ПОЛЯ С ОТНОШЕНИЕМ $I$
}

\author{
А. М. Рахматуллаев, У.А. Розиков
}

\begin{abstract}
Вводятся понятия гиббсовской меры с соответствующим потенциалом с отношением $I$ (где $I$ - подмножество множества $\{1,2, \ldots, k\}$ ), марковского случайного поля с памятью $I$ и мера с отношением $I$. Доказано, что эти три понятия эквивалентны.

Библиография: 13 названий.
\end{abstract}

1. Введение. В конце 60-х годов в теорию вероятностей был введен новый тип стохастического процесса, названньй марковским случайньм полем. Такие процессы первоначально возникли в статистической физике, но понятно, что эти процессы являются естественным обобщением марковских процессов, в которых временной индекс заменен на пространственньй.

Основные положения теории марковских случайных полей можно найти у Престона в [1], Лиггета в [2], Мальшева, Минлоса в [3], у Синая в [4].

Определение гиббсовской меры (на конечном подмножестве $Z^{\nu}$ ) восходит к классической работе Гиббса [5]. Марковские случайные поля (на $Z^{\nu}$ ) были впервые введены Добрушиным [6]. Известно, что на конечных графах классы марковских случайных полей и гиббсовских мер с потенциалом ближайшего соседа совпадают (см. например [1], [7]).

В книге [1] на конечном графе введены понятия гиббсовского состояния с потенциалом ближайшего соседа, марковского случайного поля, состояния ближайшего соседа и доказано, что эти три определения эквивалентны.

В настоящей статье вводятся понятия гиббсовской меры с соответствующим потенциалом с отношением $I$ (где $I$ - подмножество множества $\{1,2, \ldots, k\}$ ), марковского случайного поля с памятью $I$ и мера с отношением $I$. В работе доказьвается, что эти три понятия эквивалентны.

Заметим, что результат работы [1] получается в частном случае, когда $I=\{1,2\}$.

2. Основные определения и формулировка результата. Пусть $\Lambda$ - конечное множество и $P(\Lambda)$ - множество всех подмножеств этого множества.

Рассмотрим семейство функций $f_{i}: \underbrace{\Lambda \times \Lambda \times \cdots \times \Lambda}_{i \text { раз }} \rightarrow\{0,1\}, i=1, \ldots,|\Lambda|$ (где $|\cdot|-$ число элементов множества), со следуюшими свойствами:

1. $f_{i}\left(x_{1}, \ldots, x_{j}, \ldots, x_{k}, \ldots, x_{i}\right)=f_{i}\left(x_{1}, \ldots, x_{k}, \ldots, x_{j}, \ldots, x_{i}\right)$ для любых $j, k=$ $1, \ldots, i$

2. $f_{i}\left(x_{1}, x_{2}, \ldots, x_{i}\right)=0$, если $\exists j, k=1, \ldots, i$ такие, что $x_{j}=x_{k}$. 
Заметим, что функция $f_{2}: \Lambda \times \Lambda \rightarrow\{0,1\}$ определяет на $\Lambda$ структуру графа, т.е. задает отношение между парами $x, y \in \Lambda$. В этом случае можно на $\Lambda$ определить структуру графа, предполагая, что $x, y \in \Lambda$ соединены ребром графа, если $f_{2}(x, y)=1$. Наоборот, если на $\Lambda$ задана структура графа, то можно построить функцию $f_{2}: \Lambda \times \Lambda \rightarrow\{0,1\}$, которая соответствует этому графу. Эту функцию можно определить следуюшим обра3ом:

$$
f_{2}(x, y)= \begin{cases}1, & \text { если } x, y \text { соединены ребром, } \\ 0 & \text { в остальных случаях }\end{cases}
$$

Таким образом, существует взаимно однозначное соответствие между графами и функциями $f_{2}$.

Все остальные функции $f_{i}, i \neq 2$, также определяют некоторые отношения между элементами множества $\Lambda$. В этом случае будем говорить, что набор $x_{1}, x_{2}, \ldots, x_{i}$ находится в соотношении $f_{i}$, если $f_{i}\left(x_{1}, x_{2}, \ldots, x_{i}\right)=1$.

Для $x \in \Lambda$ определим

$$
\partial_{i} x=\bigcup_{\left\{y_{1}, y_{2}, \ldots, y_{i-1}\right\} \subset \Lambda \backslash\{x\}: f_{i}\left(y_{1}, y_{2}, \ldots, y_{i-1}, x\right)=1}\left\{y_{1}, y_{2}, \ldots, y_{i-1}\right\} .
$$

Введем обозначения для $A \in P(\Lambda)$ :

$$
\partial_{i} A=\left(\bigcup_{x \in A} \partial_{i} x\right) \backslash A
$$

Пусть $I \subset\{1,2, \ldots,|\Lambda|\}$, где $|\cdot|$ - число элементов множества. Обозначим

$$
\partial_{I} A=\bigcup_{i \in I} \partial_{i} A, \quad \partial_{I} x=\bigcup_{i \in I} \partial_{i} x .
$$

Непустое подмножество $B \in P(\Lambda)$ назовем $i$-симплексом, если для любого набора $y_{1}, y_{2}, \ldots, y_{i} \in B\left(y_{j} \neq y_{k}\right.$, если $\left.j \neq k\right) f_{i}\left(y_{1}, y_{2}, \ldots, y_{i}\right)=1$.

Функция $V: P(\Lambda) \rightarrow R$ называется потенииалом на $\Lambda$, если $V(\varnothing)=0$.

Пусть $V$ - потенциал на $\Lambda$. Тогда функция $J_{V}: P(\Lambda) \rightarrow \mathbb{R}$, определяемая соотношением

$$
J_{V}(A)=\sum_{X \subset A}(-1)^{|A \backslash X|} V(X),
$$

назьвается потенииалом взаимодействия, соответствующим $V$.

Заметим, что для любого $A \in P(\Lambda)$

$$
V(A)=\sum_{B \subset A} J_{V}(B) .
$$

Потенциал $V$ назовем потенииал с отношением $i$, если $J_{V}(A) \neq 0$, лишь когда $A$ есть $i$-симплекс. Пусть $V_{i}$ - потенциал на $\Lambda$ с отношением $i$. Обозначим $V_{I}=\sum_{i \in I} V_{i}$. Этот потенциал назовем потенциалом с отношением $I$.

Заметим, что

$$
J_{V_{I}}(A)=\sum_{X \subset A}(-1)^{|A \backslash X|} V_{I}(X)=\sum_{X \subset A}(-1)^{|A \backslash X|} \sum_{i \in I} V_{i}(X)=\sum_{i \in I} J_{V_{i}}(A) .
$$


Из этого равенства видно, что $J_{V_{I}}(A)=0$, если $A$ не является $i$-симплексом для любого $i \in I$.

Элементы множества $P(\Lambda)$ назьваются конфигураииями. Поскольку $P(\Lambda)$ - конечное множество, вероятностную меру на $\Lambda$ можно рассматривать как функцию $\mu: P(\Lambda)$ $\rightarrow \mathbb{R}$, где $\mathbb{R}$ - вещественная прямая, обладающая свойствами:

1) $\mu(A)>0$ для всех $A \in P(\Lambda)$;

2) $\sum_{A \subset \Lambda} \mu(A)=1$.

Пусть $M(\Lambda)$ обозначает множество всех вероятностных мер на $P(\Lambda)$.

Рассмотрим на $P(\Lambda)$ следующие вероятностные меры.

1) Мера Гиббса. Мера Гиббса на $P(\Lambda)$ с потенциалом $V$ определяется по формуле

$$
\mu(A)=Z^{-1} \exp V(A) \quad \text { для всех } A \in P(\Lambda),
$$

где $Z$ - нормируюшая константа.

2) Марковское случайное поле с памятью I. Меру $\mu$ назовем марковским случайньм полем с памятью $I$, если

а) $\mu(A)>0$ для любого $A \in P(\Lambda)$;

б) пусть $x \notin A \subset \Lambda$; тогда условная вероятность (по отношению к $\mu$ ) того, что конфигурация содержит $x$, при условии, что конфигурация на $\Lambda \backslash x$ равна $A$, совпадает с условной вероятностью того, что конфигурация содержит $x$, при условии, что конфигурация на $\partial_{I} x$ есть $A \cap \partial_{I} x$.

Пусть $\bar{A}$ - множество всех конфигураций, которые содержат $x$ и на $\Lambda \backslash x$ (ради краткости обозначим $\{x\}$ через $x$ ) совпадают с $A$, т.е. $\bar{A}=\{A \cup x\} ; \bar{B}$ - множество всех конфигураций, которые на $\Lambda \backslash x$ совпадают с $A$, т.е. $\bar{B}=\{A, A \cup x\} ; \bar{A}$ - множество всех конфигураций, которые содержат $x$, при условии, что конфигурация на $\partial_{I} x$ есть $A \cap \partial_{I} x$, т.е.

$$
\overline{\bar{A}}=\bigcup_{B \subset \Lambda \backslash\left(x \cup \partial_{I} x\right)}\left\{\left(A \cap \partial_{I} x\right) \cup x \cup B\right\} ;
$$

$\overline{\bar{B}}$ - множество всех конфигураций, которые на $\partial_{I} x$ совпадают с $A \cap \partial_{I} x$, т.е.

$$
\overline{\bar{B}}=\bigcup_{B \subset \Lambda \backslash\left(x \cup \partial_{I} x\right)}\left\{\left(A \cap \partial_{I} x\right) \cup B, \quad\left(A \cap \partial_{I} x\right) \cup x \cup B\right\} .
$$

После введенных обозначений условие б) можно переписать в следующем виде:

$$
\mu(\bar{A} \mid \bar{B})=\mu(\overline{\bar{A}} \mid \overline{\bar{B}})
$$

т.e.

$$
\frac{\mu(A \cup x)}{\mu(A \cup x)+\mu(A)}=\frac{\sum_{B \subset \Lambda \backslash\left(\partial_{I} x \cup x\right)} \mu\left(\left(A \cap \partial_{I} x\right) \cup x \cup B\right)}{\sum_{B \subset \Lambda \backslash\left(\partial_{I} x \cup x\right)} \mu\left(\left(A \cap \partial_{I} x\right) \cup x \cup B\right)+\mu\left(\left(A \cap \partial_{I} x\right) \cup B\right)} .
$$

3) Мера с отношением $I$. Меру $\mu$ назовем с отношением $I$, если

а) $\mu(A)>0$ для любого $A \in P(\Lambda)$;

б) если $x \notin A \subset \Lambda$, то

$$
\frac{\mu(A \cup x)}{\mu(A)}=\frac{\mu\left(\left(A \cap \partial_{I} x\right) \cup x\right)}{\mu\left(A \cap \partial_{I} x\right)} .
$$


Заметим, что это равенство эквивалентно равенству

$$
\frac{\mu(A \cup x)}{\mu(A \cup x)+\mu(A)}=\frac{\mu\left(\left(A \cap \partial_{I} x\right) \cup x\right)}{\mu\left(\left(A \cap \partial_{I} x\right) \cup x\right)+\mu\left(A \cap \partial_{I} x\right)} \quad \text { для любого } x \notin A \subset \Lambda .
$$

Основным результатом работы является

ТЕОрема 1. Для меры $\mu \in M(\Lambda)$ следующие утвержсдения әквивалентны:

i) $\mu-$ гиббсовская мера с потенциалом $V_{I}$;

ii) $\mu$-марковское случайное поле с памятью I;

iii) $\mu$-мера с отношением $I$.

Заметим, что при $I=\{1,2\}$ из этой теоремы следует теорема 1.1 из [1, с. 14].

3. Доказательство теоремы 1. ii) $\Longrightarrow$ iii). Пусть $\mu$-марковское случайное поле с памятью $I$, т.е. для любого $x \notin A \subset \Lambda$ выполняется равенство (2). Поскольку выражение в правой части равенства (2) не меняется при замене $A$ на $A \cap \partial_{I} x$, получим, что выполняется равенство (3), т.е. $\mu$ является мерой с отношением $I$.

iii) $\Longrightarrow$ ii $)$. Пусть $\mu$ мера с отношением $I$. Тогда для $x \notin A \subset \Lambda$ и $B \subset \Lambda \backslash\left(\partial_{I} x \cup x\right)$ получим

$$
\begin{aligned}
& \frac{\mu\left(\left(\left(A \cap \partial_{I} x\right) \cup B\right) \cup x\right)}{\mu\left(\left(A \cap \partial_{I} x\right) \cup B\right)}=\frac{\mu\left(\left(\left(\left(A \cap \partial_{I} x\right) \cup B\right) \cap \partial_{I} x\right) \cup x\right)}{\mu\left(\left(\left(A \cap \partial_{I} x\right) \cup B\right) \cap \partial_{I} x\right)} \\
& \quad=\frac{\left.\mu\left(\left(A \cap \partial_{I} x\right) \cap \partial_{I} x\right) \cup\left(B \cap \partial_{I} x\right) \cup x\right)}{\mu\left(\left(\left(A \cap \partial_{I} x\right) \cap \partial_{I} x\right) \cup\left(B \cap \partial_{I} x\right)\right)}=\frac{\mu\left(\left(A \cap \partial_{I} x\right) \cup x\right)}{\mu\left(A \cap \partial_{I} x\right)}=\frac{\mu(A \cup x)}{\mu(A)} .
\end{aligned}
$$

Откуда $\mu(A) \mu\left(\left(\left(A \cap \partial_{I} x\right) \cup B\right) \cup x\right)=\mu(A \cup x) \mu((A \cap \partial x) \cup B)$.

Суммируя по $B \subset \Lambda \backslash(\partial \cup x)$, получим (2).

i) $\Longrightarrow$ ii). В силу ii) $\Longrightarrow$ iii) достаточно показать, что $\mu$-мера с отношением $I$. Если $x \notin A \subset \Lambda$, то

$$
\frac{\mu(A \cup x)}{\mu(A)}=\exp \left\{V_{I}(A \cup x)-V_{I}(A)\right\} .
$$

Рассмотрим

$$
\begin{aligned}
V_{I}(A \cup x)-V_{I}(A) & =\sum_{B \subset A \cup x} J_{V_{I}}(B)-\sum_{B \subset A} J_{V_{I}}(B)=\sum_{B \subset A} J_{V_{I}}(B \cup x) \\
& =\sum_{B \subset A} \sum_{I \in I} J_{V_{i}}(B \cup x)=\sum_{i \in I}\left(\sum_{B \subset A} J_{V_{i}}(B \cup x)\right) .
\end{aligned}
$$

Так как $V_{i}$-потенциал с отношением $i$, т.е. $J_{V_{i}}(B \cup x) \neq 0$ только в случае, если $B \cup x-$ $i$-симплекс, то $B \subset \partial_{I} x=\bigcup_{i \in I} \partial_{i} x$. Следовательно, $B \cap \partial_{I} x=B$. Откуда следует

$$
\begin{aligned}
\sum_{i \in I}\left(\sum_{B \subset A} J_{V_{i}}(B \cup x)\right) & =\sum_{i \in I}\left(\sum_{B \subset A} J_{V_{i}}\left(\left(B \cap \partial_{I} x\right) \cup x\right)\right) \\
& =V_{I}\left(\left(A \cap \partial_{I} x\right) \cup x\right)-V_{I}\left(A \cap \partial_{I} x\right) .
\end{aligned}
$$


Следовательно,

$$
\frac{\mu(A \cup x)}{\mu(A)}=\frac{\mu\left(\left(A \cap \partial_{I} x\right) \cup x\right)}{\mu\left(A \cap \partial_{I} x\right)} .
$$

Значит, $\mu$ - мера с отношением $I$.

iii) $\Longrightarrow$ i). Пусть $\mu$-мера с отношением $I$. Докажем, что существует единственньй потенциал $V_{I}$ такой, что $\mu$ является гиббсовской мерой с потенциалом $V_{I}$.

Потенщиал $V_{I}$ определим следующим образом:

$$
V_{I}(A)=\log \left\{\frac{\mu(A)}{\mu(\varnothing)}\right\} \quad \text { для всех } A \in P(\Lambda) .
$$

Из этого равенства видно, что $\mu$ является гиббсовской мерой с потенциалом $V_{I}$ и этот потенциал единственен.

Теперь проверим, что $V_{I}$ - потенциал с отношением $I$.

Пусть $A \in P(\Lambda)$ не является $i$-симплексом для произвольного $i \in I$. Тогда найдутся такие пары $x, y \in A$, что $f_{i}\left(x, x_{2}, \ldots, x_{i-2}, y\right)=0$ для любого $i \in I$ и для любого набора $x_{2}, \ldots, x_{i-2} \in \Lambda, x_{j} \neq x_{k}, j \neq k$.

Положим $B=A \backslash x \backslash y$. Если $X \subset B$, то в силу того, что $\mu$-мера с отношением $I$, получим

$$
\begin{aligned}
\frac{\mu(X \cup x \cup y)}{\mu(X \cup x)} & =\frac{\mu\left(\left((X \cup x) \cap \partial_{I} y\right) \cup y\right)}{\mu\left((X \cup x) \cap \partial_{I} y\right)} \\
& =\frac{\mu\left(\left(X \cap \partial_{I} y\right) \cup\left(x \cap \partial_{I} y\right) \cup y\right)}{\mu\left(\left(X \cap \partial_{I} y\right) \cup\left(x \cap \partial_{I} y\right)\right)}=\frac{\mu\left(\left(X \cap \partial_{I} y\right) \cup y\right)}{\mu\left(X \cap \partial_{I} y\right)}=\frac{\mu(X \cup y)}{\mu(X)} .
\end{aligned}
$$

Следовательно,

$$
V_{I}(X \cup x \cup y)-V_{I}(X \cup x)-V_{I}(X \cup y)+V_{I}(X)=0 .
$$

Теперь вычислим $J_{V}(A)$, где $A \in P(\Lambda)$ не является $i$-симплексом для любого $i \in I$ :

$$
\begin{aligned}
J_{V_{I}}(A)= & \sum_{B \subset A}(-1)^{|A \backslash \bar{B}|} V_{I}(\bar{B}) \\
= & \sum_{X \subset B}\left\{(-1)^{|A \backslash X \cup x \cup y|} V_{I}(X \cup x \cup y)+(-1)^{|A \backslash X \cup x|} V_{I}(X \cup x)\right. \\
& \left.+(-1)^{|A \backslash X \cup y|} V_{I}(X \cup y)+(-1)^{|A \backslash X|} V_{I}(X)\right\} \\
= & \sum_{X \subset B}(-1)^{|A \backslash X|}\left\{V_{I}(X \cup x \cup y)-V_{I}(X \cup x)-V_{I}(X \cup y)+V_{I}(X)\right\}=0 .
\end{aligned}
$$

Значит, $V_{I}$ - потенциал с отношением $I$. Теорема доказана.

4. Критерий для потенциала. Следующая теорема дает критерий того, когда потенциал является потенциалом с отношением $I$.

ТЕОРема 2. Потенииал $V$ на $\Lambda$ является потенциалом с отношением I тогда и только тогда, когда для любой пары $x, y \in \Lambda$, для которой $f_{i}\left(x, x_{2}, \ldots, x_{i-2}, y\right)=0$ для любого набора $x_{2}, \ldots, x_{i-2} \in \Lambda, x_{j} \neq x_{k}, j \neq k, i \in I, u$ для любого множества $X \subset \Lambda \backslash(x \cup y)$ имеет место равенство

$$
V(X \cup x \cup y)-V(X \cup x)-V(X \cup y)+V(X)=0 .
$$


ДокАЗАТЕЛЬСтво. Необходимость. Если условие теоремы выполняется, то из доказательства теоремы 1 следует, что $V$ является потенциалом с отношением $I$.

Достаточность. Пусть $V$ - потенциал с отношением $I$, а $x, y \in \Lambda$ удовлетворяют условиям теоремы. Если $B \subset X$, то с помощюю вычислений, аналогичных тем, что были проделаны в доказательстве теоремы 1, получаем, что

$$
J_{V}(B \cup x \cup y)=\sum_{Y \subset B}(-1)^{|B \backslash Y|}\{V(Y \cup x \cup y)-V(Y \cup x)-V(Y \cup y)+V(Y)\} .
$$

Поскольку $f_{i}\left(x, x_{2}, \ldots, x_{i-1}, y\right)=0$ для любого набора $x_{2}, \ldots, x_{i-2} \in B \backslash x \backslash y$, то $B \cup x \cup y$ не является $i$-симплексом для любого $i \in I$. Следовательно, $J_{V}(B \cup x \cup y)=0$, откуда для всех $B \subset X$ имеем

$$
\sum_{Y \subset B}(-1)^{|B \backslash Y|}\{V(Y \cup x \cup y)-V(Y \cup x)-V(Y \cup y)+V(Y)\}=0 .
$$

Поэтому

$$
\begin{aligned}
V(X \cup x \cup y)-V(X \cup x)-V(X \cup y)+V(X) \\
=\sum_{B \subset X \cup x \cup y} J_{V}(B)-\sum_{B \subset X \cup x} J_{V}(B)-\sum_{B \subset X \cup y} J_{V}(B)+\sum_{B \subset X} J_{V}(B) \\
=\sum_{B \subset X} J_{V}(B \cup x \cup y) \\
=\sum_{B \subset X} \sum_{Y \subset B}(-1)^{|B \backslash Y|}\{V(Y \cup x \cup y)-V(Y \cup x)-V(Y \cup y)+V(Y)\}=0 .
\end{aligned}
$$

Теорема доказана.

5. Примеры. 1. Пусть $\Lambda=\{1,2, \ldots, n\}$ - конечное множество. Рассмотрим функции

$$
\begin{aligned}
f_{1}(x)= & \left\{\begin{array}{ll}
1, & \text { если } x \text { четно, } \\
0, & \text { если } x \text { нечетно, }
\end{array} \quad f_{2}(x, y)= \begin{cases}1, & \text { если }|x-y|=1, \\
0 & \text { в остальных случаях, }\end{cases} \right. \\
& f_{3}(x, y, z)= \begin{cases}1, & \text { если }|x-y|=|x-z|=|y-z|=1, \\
0 & \text { в остальных случаях. }\end{cases}
\end{aligned}
$$

Потенциал $V$ определим следуюшим образом:

$$
\begin{gathered}
V(A)=J_{1} \sum_{x \in A: f_{1}(x)=1} \sigma(x)+J_{2} \sum_{\{x, y\} \subset A: f_{2}(x, y)=1} \sigma(x) \sigma(y) \\
+J_{3} \sum_{\{x, y, z\} \subset A: f_{3}(x, y, z)=1} \sigma(x) \sigma(y) \sigma(z),
\end{gathered}
$$

где $\sigma(x)= \pm 1$ для любого $x \in \Lambda$. Заметим, что в этом случае

$$
J_{V}(A)= \begin{cases}J_{1} \sigma(x), & \text { если } A=\{x\}, f_{1}(x)=1, \\ J_{2} \sigma(x) \sigma(y), & \text { если } A=\{x, y\}, f_{2}(x, y)=1, \\ J_{3} \sigma(x) \sigma(y) \sigma(z), & \text { если } A=\{x, y, z\}, f_{3}(x, y, z)=1, \\ 0 & \text { в остальных случаях. }\end{cases}
$$


Таким образом, построенньй потенциал является потенциалом с отношением $I=\{1,2,3\}$ $\subset\{1,2, \ldots, n\}$.

2. Пусть $\Lambda$ - команда спортсменов, состоящая из 14 футболистов и 6 теннисистов.

Рассмотрим функции

$$
\begin{gathered}
f_{1}(x)=\left\{\begin{array}{l}
1, \text { если } x-\text { теннисист, } \\
0, \text { если } x-\text { футболист, }
\end{array}\right. \\
f_{2}(x, y)= \begin{cases}1, & \text { если } x, y-\text { теннисисты, } x \neq y, \\
0 & \text { в остальных случаях, }\end{cases} \\
f_{11}\left(x_{1}, x_{2}, \ldots, x_{11}\right)= \begin{cases}1, & \text { если } x_{1}, \ldots, x_{11}-\text { различные футболисты, } \\
0 & \text { в остальных случаях. }\end{cases}
\end{gathered}
$$

Функцию $\sigma: \Lambda \rightarrow\{-1 ; 1\}$ определим следующим образом:

$$
\sigma(x)= \begin{cases}1, & \text { если } x \text { вьиграл, } \\ -1, & \text { если } x \text { проиграл. }\end{cases}
$$

Тогда потенциал

$$
\begin{aligned}
V(A)= & \sum_{x \in A: f_{1}(x)=1} \sigma(x)+\sum_{x, y \in A: f_{2}(x, y)=1}(\sigma(x)+\sigma(y)) \\
& +\sum_{x_{1}, \ldots, x_{11} \in \Lambda: f\left(x_{1}, \ldots, x_{11}\right)=1}\left(\sigma\left(x_{1}\right)+\cdots+\sigma\left(x_{11}\right)\right) .
\end{aligned}
$$

Заметим, что построенный потенциал является потенциалом с отношением $I=\{1,2,11\}$ $\subset\{1,2, \ldots, 20\}$.

ЗАмЕчАния. Определение гиббсовской меры и другие определения настоящей работы проведены на конечных множествах. Структура графа на $\Lambda$ не имеет значения в наших определениях. Поэтому эти определения являются рабочими и для достаточно широких классов моделей физики, химии, социологии, биологии и других разделов науки.

В книге [1] определены гиббсовское состояние с потенциалом ближайшего соседа, марковское случайное поле и состояние ближайшего соседа на счетных графах и доказано, что все эти определения эквивалентны.

В случае счетного графа возникает вопрос об описании предельного состояния Гиббса. В некоторых случаях это состояние оказьвается не единственным. В случае неединственности предельного гиббсовского состояния говорят, что имеет место фазовый переход. Вообще говоря, множество предельных гиббсовских состояний для данного потенциала на счетном графе может оказаться континуальным.

Определению и изучению гиббсовских мер с потенциалом $V_{I}$ на счетных множествах будут посвящены следующие работы авторов. 


\section{СПИСОК ЦИТИРОВАННОЙ ЛИТЕРАТУРЫ}

[1] Престон К. Гиббсовские состояния на счетных множествах. М.: Мир, 1977.

[2] Лиггетт Т. Марковские процессы с локальным взаимодействием. М.: Мир, 1989.

[3] Малышев В.А., Минлос Р. А. Гиббсовские случайные поля. М.: Наука, 1985.

[4] Синай Я. Г. Теория фазовых переходов. М.: Наука, 1980.

[5] Gibbs W. Elementary Principles of Statistical Mechanics: Yale University, 1902.

[6] Добрушин Р. Л. Описание случайного поля при помощи условных вероятностей и условия его регулярности // ТВП. 1968. № 2. С. 201-229.

[7] Spitzer F. Markov random fields and Gibbs ensembles // Ann. Math. Monthly. 1971. V. 78. P. 142-154.

[8] Добрушин Р. Л. Исследование гиббсовских состояний для трехмерных решеточных систем // ТВП. 1973. T. XVIII. №2. C. 261-279.

[9] Ганиходжаев Н. Н., Розиков У. А. Описание периодических крайних гиббсовских мер некоторых моделей на дереве Кэли // ТМФ. 1997. Т. 111. №1. С. 109-117.

[10] Розиков У. А. Структура разбиений группового представления дерева Кэли и их применения для описания периодических распределений Гиббса // ТМФ. 1997. Т. 112. № 1. С. 170-176.

[11] Розиков У. А. Описание предельных гиббсовских мер $\lambda$-моделей на решетке Бете // Сиб. матем. ж. 1998. Т. 39. № 2. С. 427-435.

[12] Розиков У. А. Построение несчетного числа гиббсовских мер неоднородной модели Изинга // ТМФ. 1999. Т. 118. №1. С. 95-104.

[13] Ганиходжаев Н. Н., Розиков У. А. О неупорядоченных фазах некоторых моделей на дереве Кэли // Матем. сб. 1999. Т. 190. № 2. С. 31-42.

Институт математики АН РУз, г. Ташкент

Поступило

E-mail: anasbek@mail.com, root@im.tashkent.su

29.01.2001 